\title{
SoFAR: Software for Fully Automatic Evaluation of Real-Time PCR Data
}

BioTechniques 34:324-332 (February 2003)

\author{
Jochen Wilhelm, Alfred \\ Pingoud, and Meinhard Hahn \\ Justus-Liebig-Universität \\ Giessen, Giessen, Germany
}

\section{INTRODUCTION}

Quantitative real-time PCR has become an important tool to determine the concentrations of nucleic acids for a variety of different purposes (13). The product accumulation is monitored during the PCR process in real time by fluorescence techniques $(2,5-8,20-23)$. These techniques combine high sensitivity with a high dynamic range for quantification without the requirement of a post-PCR analysis. The fluorescence signal curves from the individual amplification reactions are used to determine $\mathrm{C}_{\mathrm{T}}$ values (i.e., the crossing points of the signal curves with an arbitrary threshold) (6). Under the condition that the threshold intersects the amplification curves in their exponential phase, the $\mathrm{C}_{\mathrm{T}}$ values determined are proportional to the logarithm of the initial copy numbers. Using the information of $\mathrm{C}_{\mathrm{T}}$ values, relative quantifications are possible with the $\Delta \Delta \mathrm{C}_{\mathrm{T}}$ method $(11,16)$. Quantitative results can be obtained when standards with known concentrations are used to determine the amplification efficiency $(14,15,18,19)$.

The software solutions provided by the manufacturers of real-time PCR machines calculate the $C_{T}$ values and, based on these, the concentrations of unknown samples, but usually these programs have shortcomings. (i) They do not use all available data of the signal curves for the calculation of the $\mathrm{C}_{\mathrm{T}}$ values, making the results sensitive to signal noise. (ii) The applied threshold value is either arbitrary [10 times the standard deviation of the signal values in the first 3-10 cycles; see LightCy- cler $^{\mathrm{TM}}$ (Roche Diagnostics, Mannheim, Germany) and ABI 7700 manuals (Applied Biosystems, Foster City, CA, USA)] or has to be chosen by the user. (iii) The applicability of the amplification curves and the validity of the threshold for quantitative evaluation are not assessed. (iv) No estimation is given for the accuracy of the quantification. In total, the available software often leads to questionable results of suboptimal accuracy and requires time and experience for data interpretation.

To circumvent the sensitive dependency of $\mathrm{C}_{\mathrm{T}}$ calculations, sigmoid functions have been employed to describe the amplification curves $(9,10)$. The functions used are symmetric, which is not necessarily the case for amplification curves. Therefore, the fitted function filters noise well but may deviate systematically, in particular in the exponential phase, yielding erroneous $\mathrm{C}_{\mathrm{T}}$ values.

Here we present software for the optimum evaluation of amplification and melting curve data of LightCycler realtime PCR experiments (22). The software can perform an adaptive background correction on the amplification curves to remove amplification-independent trends and allows the calculation of the effective noise. It identifies the exponential phases automatically and uses the fit of an amplification function through all points within this phase to calculate the $\mathrm{C}_{\mathrm{T}}$ values. The optimal threshold is automatically adjusted by minimizing the error of the calibration curve. The adaptive smoothing of the melting curve raw data allows the calculation of unambiguous melting peaks. A temperature 
Table 1. Equations

\begin{tabular}{|c|c|c|}
\hline No. & Formula & Description \\
\hline 1 & $\mathrm{~F}_{\mathrm{c}}^{\mathrm{m}}=\sum_{\mathrm{i}=1}^{\mathrm{n}} \mathrm{F}_{\mathrm{c}, \mathrm{i}}^{\mathrm{r}}=\sum_{\mathrm{i}=1}^{\mathrm{n}} \mathrm{a}_{\mathrm{c}, 1} \cdot \mathrm{F}_{\mathrm{i}}^{\mathrm{r}}$ & $\begin{array}{l}\text { Fluorescence of multicolor measurements } \\
\mathrm{F}_{\mathrm{c}}^{\mathrm{m}} \text { : measured total fluorescence intensity in channel } \mathrm{c} ; \mathrm{i} \text { : index } \\
\text { variable; } \mathrm{n} \text { : number of fluorophores (= number of channels); } \\
\mathrm{F}_{\mathrm{c}, \mathrm{i}}^{\mathrm{r}} \text { : real fluorescence intensity of fluorophore i measured in } \\
\text { channel } \mathrm{c} ; \mathrm{a}_{\mathrm{c}, 1} \text { : parameter matrix defining the fractions of the } \\
\text { signal of each fluorophor } \mathrm{i} \text { in each channel } \mathrm{c} ; \mathrm{F}_{\mathrm{i}}^{\mathrm{r}} \text { ( (unknown) real } \\
\text { fluorescence intensity of fluorophor i measured in the channel, } \\
\text { including the maximum of the emission spectrum of the } \\
\text { fluorophor. }\end{array}$ \\
\hline 2 & $B(x)=a \cdot\left(1-e^{-b x}\right)+c$ & $\begin{array}{l}\text { Saturation function for background correction } \\
B(x) \text { : background function; } x \text { : cycle number; a: saturation value; } \\
\text { b: slope parameter; c: signal-offset }\end{array}$ \\
\hline 3 & $A(x)=N_{\max } \cdot \frac{1}{1+e^{-r\left(x_{T}-x\right)}}$ & $\begin{array}{l}\text { Sigmoid amplification function } \\
A(x) \text { : amplification function; } x \text { : amplification cycle; } N_{\text {max }} \text { : plateau } \\
\text { value; } r \text { : efficiency parameter [ } r \text { adjusts the slope in the turning } \\
\left.\text { point }\left(x=x_{T}\right)\right] ; x_{T} \text { : cycle of the turning point of the curve }\end{array}$ \\
\hline 4 & $\mathrm{v}_{\mathrm{T}}=\mathrm{e}^{1 / 2(\ln (\min )+\ln (\max ))}$ & $\begin{array}{l}\text { Logarithmic mean } \\
\mathrm{v}_{\mathrm{T}} \text { : threshold value; min: lower signal limit of the common } \\
\text { exponential phase; max: upper signal limit of the common } \\
\text { exponential phase }\end{array}$ \\
\hline 5 & $\mathrm{~N}_{0}=10^{\frac{\mathrm{C}_{T}-\mathrm{b}}{\mathrm{m}}}$ & $\begin{array}{l}\text { Initial concentration } \\
\mathrm{N}_{0} \text { : initial concentration; } \mathrm{C}_{\mathrm{T}} \text { : determined } \mathrm{C}_{\mathrm{T}} \text { value; } \mathrm{b} \text { : intercept of } \\
\text { the calibration curve; } \mathrm{m} \text { : slope of the calibration curve }\end{array}$ \\
\hline 6 & $\overline{\mathrm{CV}}=\frac{1}{\mathrm{n}} \sum \frac{\mathrm{N}_{0}^{\mathrm{calc}}-\mathrm{N}_{0}^{\mathrm{std}}}{\mathrm{N}_{0}^{\mathrm{calc}}}$ & $\begin{array}{l}\text { Mean coefficient of variation } \\
\overline{\mathrm{CV}} \text { : mean coefficient of variation; } \mathrm{n} \text { : number of standards; } \mathrm{N}_{0}^{\text {calc }} \text { : } \\
\text { calculated initial standard concentrations; } \mathrm{N}_{0}^{\text {std }} \text { : defined initial } \\
\text { standard concentrations }\end{array}$ \\
\hline 7 & $S_{y, x}^{C_{T}}=\sqrt{\frac{1}{n(n-2)}\left(n \Sigma y^{2}-(\Sigma y)^{2}-\frac{(n \Sigma x y-(\Sigma x)(\Sigma y))}{n \Sigma x^{2}-(\Sigma x)^{2}}\right)}$ & $\begin{array}{l}\text { Standard error of } \mathrm{C}_{\mathrm{T}} \text { values } \\
\mathrm{S}_{\mathrm{y}, \mathrm{x}}^{\mathrm{C}_{\mathrm{T}}} \text { : standard error of the estimated } \mathrm{y} \text { values for the } \mathrm{x} \text { values of } \\
\text { the regression analysis, calculated for the } \mathrm{C}_{\mathrm{T}} \text { values; } \mathrm{n} \text { : number } \\
\text { of standards; } \mathrm{x} \text { : logarithms of the initial standard concentrations } \\
\text { as used for the regression analysis of the calibration curve; } \\
\mathrm{y} \text { : determined } \mathrm{C}_{\mathrm{T}} \text { values of the standards }\end{array}$ \\
\hline 8 & $S_{y, x}^{N_{0}}=\left(\varepsilon^{S_{y, x}^{C_{T}}}\right)-1=\left(10^{\left(m^{-1}\right) \cdot S_{y, x}^{C_{T}}}\right)-1$ & $\begin{array}{l}\text { Standard error of initial concentrations } \\
S_{y, x}^{N_{0}}: \text { standard error of the estimated } y \text { values for the } x \text { values of } \\
\text { the regression analysis, calculated for the initial concentrations; } \\
S_{y, x}^{C_{T}} \text { : standard error of } C_{T} \text { values (equation } 7 \text { ); } \varepsilon \text { : amplification } \\
\text { efficiency as determined from the slope of the calibration curve; } \\
m \text { : slope of the calibration curve }\end{array}$ \\
\hline 9 & $y(T)=\sum_{i=1}^{n} h_{i} \cdot e^{-\left(\frac{T-\mu_{i}}{\sigma_{i}}\right)^{2}}$ & $\begin{array}{l}\text { Peak function for melting curve analysis } \\
y(T): \text { peak function; } T \text { : temperature; i: index variable; } n \text { : number of } \\
\text { peaks; } h_{i} \text { : height of peak } i ; \mu_{i}: \text { center of peak } i ; \sigma_{i}: \text { width of peak } i .\end{array}$ \\
\hline 10 & $\mathrm{~A}(\mathrm{~h}, \sigma)=\mathrm{h} \cdot \sigma \cdot \sqrt{\pi}$ & $\begin{array}{l}\text { Peak area } \\
A(h, \sigma) \text { : peak function; } h \text { : height of the peak; } \sigma: \text { width of the peak }\end{array}$ \\
\hline
\end{tabular}


quench correction can be performed on melting curve data for a precise calculation of melting peak areas, as needed for competitive quantifications or quantitative SNP analyses.

The software is comprehensive, easy to use, and helps even the non-expert to obtain reliable results in minimal time.

\section{MATERIALS AND METHODS}

\section{Data Files}

For the purpose of demonstration, the data files distributed with the Software Version 3 of the LightCycler PCR System were analyzed.

\section{Color Compensation for Multicolor Measurements}

In each detection channel, the measured fluorescence signal of a mixture of fluorophores, including the donor fluorophore, is the sum of individual signals from each fluorophore as given in Table 1, equation 1 . The parameter matrix "a", defining the individual fractions of the signal for each fluorophore and channel, is determined in a calibration run with the Roche Color Compensation Kit. Knowing matrix "a", the equation system can be solved for the individual signals.

\section{Evaluation Procedure for Quantifications}

The amplification-independent trends in the signal are eliminated by subtracting a saturation function (Table 1 , equation 2) whose parameters are determined for each signal curve individually from the data before the point of maximum bending ( $\mathrm{c}_{\mathrm{bend}}$ in Figure 1a) by a least squares fit using the Levenberg-Marquard algorithm (12). Briefly, this algorithm performs a sequence of linear optimizations (finding the minimum of a 1-D function) to determine the minimum of the error function $\chi^{2}$ (sum of squared residuals between the measured and predicted values). Starting with an initial estimate for the parameters (a), the linear optimization is done in the direction of the steepest gradient $\delta \mathbf{a} / \delta \chi^{2}$. The procedure is re- peated until a given tolerance for the change in $\chi^{2}$ is reached. Then, the signal noise is calculated from the standard deviation of the points before $\mathrm{c}_{\text {bend }}$ (Figure 1a).

The logarithms of the backgroundcorrected time courses are used to estimate the detectable exponential phase. The global extremes of their second derivatives at the cycle numbers $c_{\text {exp,min }}$ and $\mathrm{c}_{\text {exp,max }}$ define the range of cycles where an exponential signal increase is detected. The starting cycle of the detectable exponential phase, $\mathrm{c}_{\mathrm{exp} \text {,min }}$, is increased, if necessary, to ensure that the smallest value used is higher than the noise. The exponential range is accepted when it comprises more than three cycles (Figure 1b).

Then, the software fits the amplification function $[\mathrm{A}(\mathrm{x})$ : Table 1 , equation
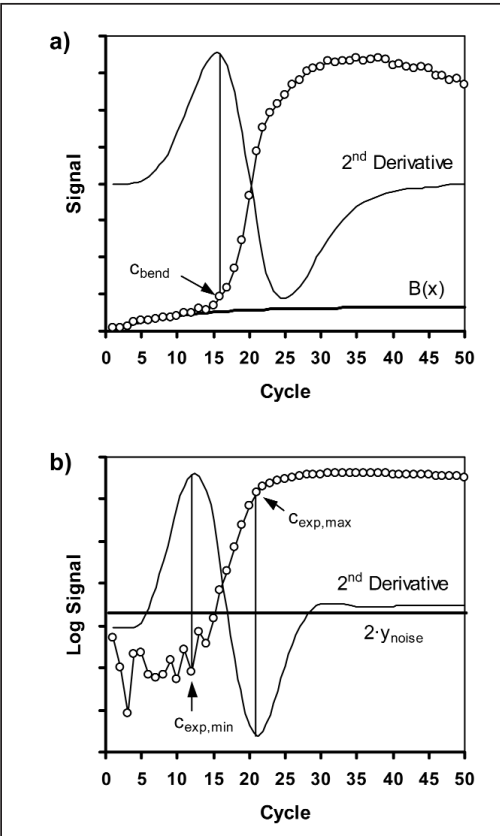

Figure 1. Background correction of amplification curves and determination of the observable exponential phase. (a) Amplification curve (circles) and its second derivative (thin line), calculated from a cubic smoothing spline through the data points. The background trend function $\mathrm{B}(\mathrm{x})$ (thick line) is extrapolated from the data points before cycle $c_{\text {bend }}-4$. For correction, the values of $\mathrm{B}(\mathrm{x})$ are subtracted from each data point. (b) Half-logarithmic representation of the corrected amplification data shown in part a (circles) and its second derivative, calculated from a cubic smoothing spline through the log data points. The points considered to be within the observable exponential phase are between $c_{\text {exp, min }}$ and $c_{\text {exp,max }}$. Data points below the noise band (thick line) are excluded.
3] to all points of each curve within that phase using the Levenberg-Marquard algorithm (12). Next, the $\mathrm{C}_{\mathrm{T}}$ value is determined for a given threshold value $\mathrm{v}_{\mathrm{T}}$ using Newton's algorithm to solve $\mathrm{A}(\mathrm{x})-\mathrm{v}_{\mathrm{T}}=0$ for $\mathrm{x}$, which starts from an initial estimate for $x$ and predicts the next, improved estimate for $\mathrm{x}$ from $\mathrm{s}(\mathrm{x})$ $=0$, where $\mathrm{s}$ is a straight line through the point $(\mathrm{x} / \mathrm{y}(\mathrm{x}))$ with the slope of $\mathrm{A}$ at $\mathrm{x}$, until $\mathrm{y}(\mathrm{x})$ is smaller than a given tolerance.

The threshold is adjusted to that value resulting in a minimal error in the calibration curve. If no standards are defined, then the threshold is set to the logarithmic mean (Table 1, equation 4) between the upper and lower signal limits of the common exponential phase of all selected samples.

The initial concentrations are calculated using Table 1, equation 5, derived
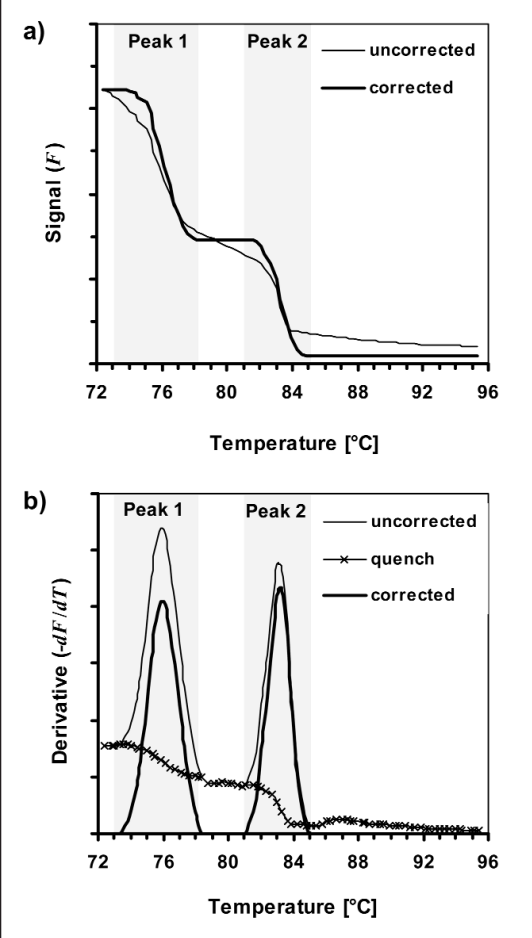

Figure 2. Temperature-dependent quench correction in melting curve analysis. (a) Plots of signal versus temperature of the data obtained for a mixture of two products with different melting points before and after correction. Steep decreases in signal correlate with the melting processes (gray boxes). Signal changes outside of the gray boxes are presumably not due to DNA melting. They are eliminated after correction. (b) Melting peak representation of the data shown in part a. Signal change, which is considered not to be due to melting of product ("quench"), is also shown. 


\begin{tabular}{|c|c|c|}
\hline Icon & Color & Meaning \\
\hline \multicolumn{3}{|c|}{ Standards } \\
\hline \multirow{2}{*}{ 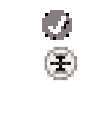 } & green & Valid \\
\hline & yellow & $\begin{array}{l}\text { Threshold value is outside the exponential phase; } \\
\mathrm{C}_{\mathrm{T}} \text { value is not calculated. }\end{array}$ \\
\hline $\mathbf{8}$ & red & No exponential phase \\
\hline \multicolumn{3}{|c|}{ Unknowns } \\
\hline t & green & Positive: $\mathrm{C}_{\mathrm{T}}$ value is calculated. \\
\hline 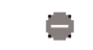 & green & Negative: no exponential phase. \\
\hline$\oplus$ & yellow & $\begin{array}{l}\text { Calculated concentration is higher than the highest } \\
\text { standard concentration. }\end{array}$ \\
\hline 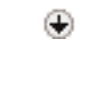 & yellow & $\begin{array}{l}\text { Calculated concentration is lower than the lowest } \\
\text { standard concentration. }\end{array}$ \\
\hline ( & yellow & $\begin{array}{l}\text { Threshold value is outside the exponential phase; } \\
\mathrm{C}_{\mathrm{T}} \text { value is not calculated. }\end{array}$ \\
\hline (6) & yellow/red & $\begin{array}{l}\text { The efficiency, calculated from the slope of the } \\
\text { exponential phase, is more than } 1.5 \text { higher than the } \\
\text { mean efficiency of the standards. }\end{array}$ \\
\hline (9) & yellow/blue & $\begin{array}{l}\text { The efficiency, calculated from the slope of the } \\
\text { exponential phase, is more than } 1.5 \text { lower than the } \\
\text { mean efficiency of the standards. }\end{array}$ \\
\hline ○ & gray & $\begin{array}{l}\text { Characterization is not possible, because of an invalid } \\
\text { calibration. }\end{array}$ \\
\hline \multicolumn{3}{|c|}{ Positive Controls } \\
\hline$\oplus$ & green & Positive: exponential phase is detectable. \\
\hline 불 & red & False negative: no exponential phase. \\
\hline \multicolumn{3}{|c|}{ Negative Controls } \\
\hline 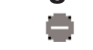 & green & Negative: no exponential phase. \\
\hline (4) & red & False positive: exponential phase is detectable. \\
\hline
\end{tabular}

from the calibration curve. The mean coefficient of variation of the standard concentrations is calculated using Table 1, equation 6. The standard error of the $\mathrm{C}_{\mathrm{T}}$ values is calculated using Table 1, equation 7. This result is used to estimate the standard error of the calculated concentrations by Table 1 , equation 8 .

The status of each result is determined by the following procedure. If the sample gives a valid exponential phase, but the threshold value does not intersect the amplification curve within this range, then the status is set to "invalid threshold". Otherwise, the status depends on the sample type (Table 2). In all cases, except for "negative" characterizations or a failed calibration, the concentrations of the samples are calculated and shown in the results table.

\section{Melting Curve Analysis}

The raw data used for melting curve analysis are the temperature-dependent signals. The raw data are smoothed by interpolation using cubic smoothing splines $(3,4,17)$. The smoothing parameter is chosen automatically depending on the number of data points. The uncorrected peak data are calculated as the negative derivative $(-d \mathrm{~F} / d \mathrm{~T})$ of the splines. This peak data are smoothed again and used for the optional temperature-dependent quench correction. The processing of the data to correct the temperature-dependent quench of fluorescence comprises several steps. An overview of the sequence of calculations is as follows (Figure 2). (i) The approximate number and positions of the peaks are determined through the local maxima in the spline for the uncorrected peak 
data. The temperature range of the peak is widened to the adjacent local minima. The signal decreases of the raw data outside of these temperature ranges are assumed not to be due to product melting. (ii) The temperature-dependent quench is calculated using the information of the approximate peak positions. Outside the peak temperature ranges, the whole signal decrease in the raw data is defined as quench, whereas, within the peak temperature ranges, product melting has to be taken into account. The melting process is approximately proportional to the signal decrease in the raw data. The quench within that range is described by the corresponding raw data, which are projected in a way that they connect the already determined quench values before and after the melting process. (iii) The preliminary corrected peak data are calculated by subtracting the quench values from the uncorrected peak data. (iv) The corrected melting curve data are calculated by integrating the corrected peak data and are then projected into the total signal decrease of the corresponding raw data. As the result, starting and
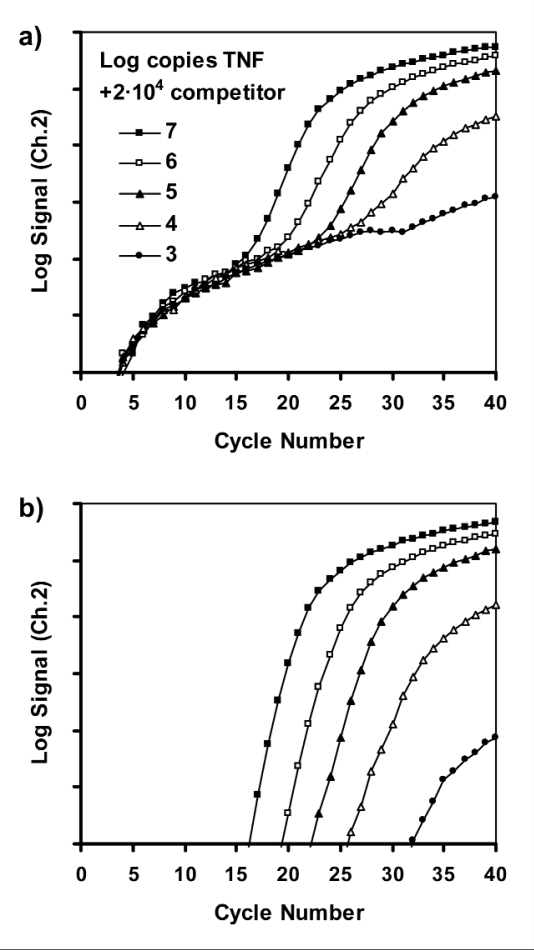

Figure 3. Background correction of kinetic PCR data. Data from "DemoQuant-DualColor". (a) Raw data acquired in channel 2 of the standards. (b) Same signal curves as in part a after background correction. ending signal values of the corrected data are the same as for the raw data. (v) In a last step, the final corrected peak data are calculated from the corrected and normalized melting curve.

Finally, the peak parameters are calculated by fitting the peak function (Table 1, equation 9) to the uncorrected or corrected peak data. The parameter $\mu_{\mathrm{i}}$ corresponds to the $\mathrm{T}_{\mathrm{m}}$. The peak areas, being proportional to the amount of product, are calculated from the parameters $h$ and $\sigma$ of the individual peaks using Table 1, equation 10.

\section{RESULTS AND DISCUSSION}

\section{Analysis}

Figure 3 shows the effect of the background correction on real-time PCR data. Without trend correction, the signal curves are increasing before their exponential phases, which can be identified

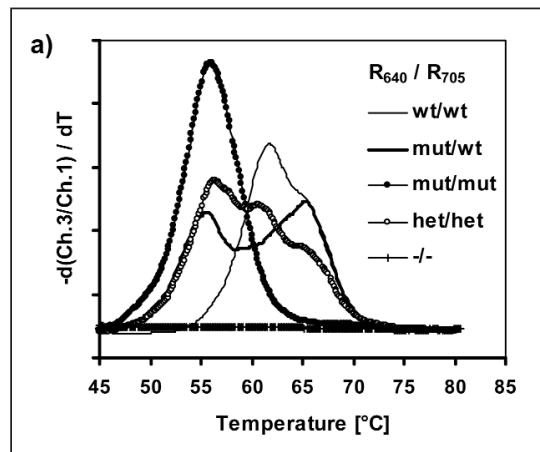

b)

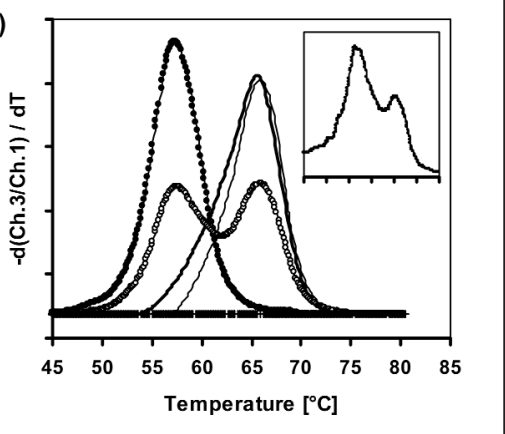

Figure 4. Color compensation. Data from "DemoMutation-DualColor". Shown are the melting peaks derived from channel 3 (second position, analyzed with the $\operatorname{Red}_{705}$-labeled probe. wt: $\frac{\mathrm{wt}}{\mathrm{wt}}$ mut: $\frac{\text { mut }}{\text { mut }}$ het: $\frac{\mathrm{wt}}{\text { mut }}$ (a) Melting peaks without color compensation. (b) Melting peak data with color compensation. The inset shows the melting peak data with color compensation, analyzed with the Roche Analysis Software LCDA Version 3.5.28 under preset adjustments (digital filter, on; degrees to average, 4; calculation method, polynomial). in the half-logarithmic plot as more or less straight segments (Figure 3a). The slope of these segments, indicating the individual amplification efficiency, increases with initial target concentration. The initial trends are eliminated after background correction and reveal longer exponential phases with more similar and higher slopes (Figure $3 b$ ).

Figure 4 illustrates the effect of color compensation, showing the results of dual-color mutation detection. The assay is described in detail by Bernard et al. (1). Briefly, two positions (codons 112/158) of the apo E locus were simultaneously analyzed using two pairs of hybridization probes. The first position was detected with a $\operatorname{Red}_{640}$-labeled probe (channel 2), the second with a Red $_{705}$-labeled probe (channel 3). The sample $\frac{\mathrm{mut}}{\mathrm{mut}} / \frac{\mathrm{wt}}{\mathrm{wt}}$ is expected to be represented by a single melting peak of the
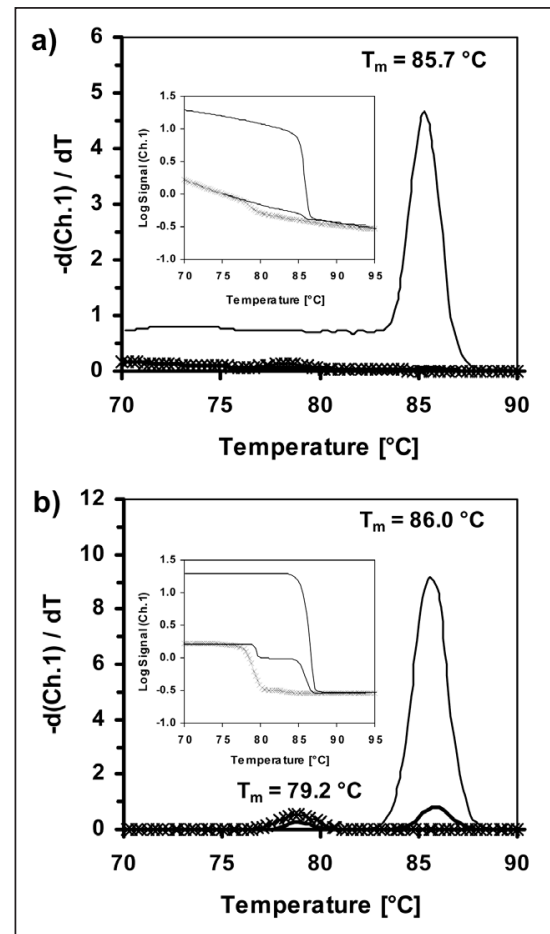

Figure 5. Increased sensitivity of melting curve analyses after temperature-dependent quench correction. Data from "DemoQuant-SYBRGreen". Shown are the data of channel 1 from "Standard 10E2" (100 target copies, thin line), "Standard 1E1" (10 target copies, thick line), and "No Template" (crossed line). Note the log scale of the abscissas in the insets. (a) Melting peak data without correction. Inset: half-logarithmic plots of the uncorrected raw data. (b) Corrected melting peak data. Inset: half-logarithmic plots of the corrected raw data. 
$\operatorname{Red}_{705}$ probe with a $T_{m}$ value of $66^{\circ} \mathrm{C}$. The sample $\frac{\mathrm{wt}}{\mathrm{mut}} / \frac{\mathrm{wt}}{\mathrm{mut}}$ should appear as two peaks with $\mathrm{T}_{\mathrm{m}}$ values of $57^{\circ} \mathrm{C}$ and $66^{\circ} \mathrm{C}$. Here, in both cases, $\operatorname{Red}_{640}$ fluorescence significantly alters the signal measured in channel 3 so that $\frac{\mathrm{mut}}{\mathrm{mut}} / \frac{\mathrm{wt}}{\mathrm{wt}}$ seems to be heterozygous in the second position and $\frac{\mathrm{wt}}{\mathrm{mut}} / \frac{\mathrm{wt}}{\mathrm{mut}}$ shows three peaks (Figure 4a). After color compensation, the sample $\frac{\text { mut }}{m u t} / \frac{\mathrm{wt}}{\mathrm{wt}}$ appears as a single peak, indicating a $\frac{\mathrm{wt}}{\mathrm{wt}}$ state at position 2 and the middle peak of $\frac{\mathrm{wt}}{\mathrm{mut}} / \frac{\mathrm{wt}}{\mathrm{mut}}$ is removed (Figure $4 \mathrm{~b}$ ). For comparison, the melting peak data calculated with the Roche LCDA software (distributed with the LightCycler system) are shown in the insert of Figure 4b: the two distinct peaks of the $\frac{\mathrm{wt}}{\mathrm{mut}} / \frac{\mathrm{wt}}{\mathrm{mut}}$ sample have unequal areas, although the amounts of sequence variants in a heterozygous sample are equal. This problem does not occur when the data are evaluated with SoFAR.

Figure 5 demonstrates the effects of the quench correction for melting curve analyses. Without correction, the melting-related signal decreases of the control and the samples with 10 target copies do not appear as melting peaks; only the sample with 100 target copies has a clear melting peak at $\mathrm{T}_{\mathrm{m}}=85.7^{\circ} \mathrm{C}$ (Figure 5a). After correction, the melting-related signal changes of all samples become apparent (Figure 5b) and can be identified unambiguously as peaks with $\mathrm{T}_{\mathrm{m}}$ values of $79.2^{\circ} \mathrm{C}$ (no template control, probably representing minor amounts of primer dimers) and $86.2^{\circ} \mathrm{C}$. The peak areas for the specific products calculated are 21.1 (100 copies) and 0.92 (10 copies).

\section{Structure, Features, and Characteristics of SoFAR}

The software consists of a single file that can be used without installation. It runs under all versions of Microsoft ${ }^{\circledR}$ Windows ${ }^{\mathrm{TM}}$ on computers with at least $500 \mathrm{MHz}$ and $32 \mathrm{MB}$ RAM. Figure 6 gives screenshots of the different forms. The main application contains a sample selection panel ("SP"), an adjustment panel ("AP") with all settings adjustable, and a client area ("CA") with several windows showing data and results.

Data files recorded by the LightCycler software (Run Version 1.20.vi to
5.10) can be analyzed. The program uses the information from the aboutfile (.ABT) and the fluorescence file (.FLO). The temperature files (.TEM) are not required. In principle, the software can be adapted to work with all other available real-time PCR systems, provided the data format is known.

After opening a file, all analyses are done automatically. Usually, no additional adjustments are needed. After opening a file, the samples are hierarchically sorted by run, sample type, and groups. All sample names are shown in a treeview representing the group organization, with a checkbox and a legend symbol for the data curves of the individual samples. The samples of several runs with the same protocol can be analyzed simultaneously. Any change made by the user will lead to an immediate update of all results.

All available information, data, and results are presented in four different sections, Run Info, Segment Data, Quantification, and Melting Curves, that consist of a selection panel section and one to three client windows. The software will only show the Quantification or Melting Curves sections if the run protocol contains a program with an appropriate analysis mode and collected dataset.

The section Run Info (a in Figure 6) contains one client window showing all general information of all runs loaded and the cycling protocol. The imported data from color compensation calibration files are listed in a drop-down selection box of the client window, where they can be selected. Each run loaded may use a different color compensation calibration dataset.

The Segment Data section has one client window for the graphical representation of any collected data. The user can select any data-containing segment to be analyzed and can define any data 


\section{Research Report}

source to be represented on both axes.

In the Quantification section (b in Figure 6), the fluorescence data and the calibration curve are shown in separate client windows. The third client window contains the results of the selected samples and of the statistical analyses. Each selected sample is additionally characterized concerning the existence of an exponential phase and the validity of the quantification, as indicated by icons in the first column of the quantification results table. Table 2 shows all possible characterizations. The calibration is not done if one of the following conditions is true: $(i)$ less than two standards with different concentrations are selected; (ii) at least one standard has no valid $\mathrm{C}_{\mathrm{T}}$ value; (iii) the exponential phases of the selected standards do not overlap; and (iv) the range of the amplification efficiencies, calculated from the slopes of the exponential phases of the standards, is larger than 0.3 , an empirically determined cut-off value. The reason for a calibration failure is shown in the status field of the quantification results window. The user can choose the data and the channel to be analyzed. The software has the options of chang-

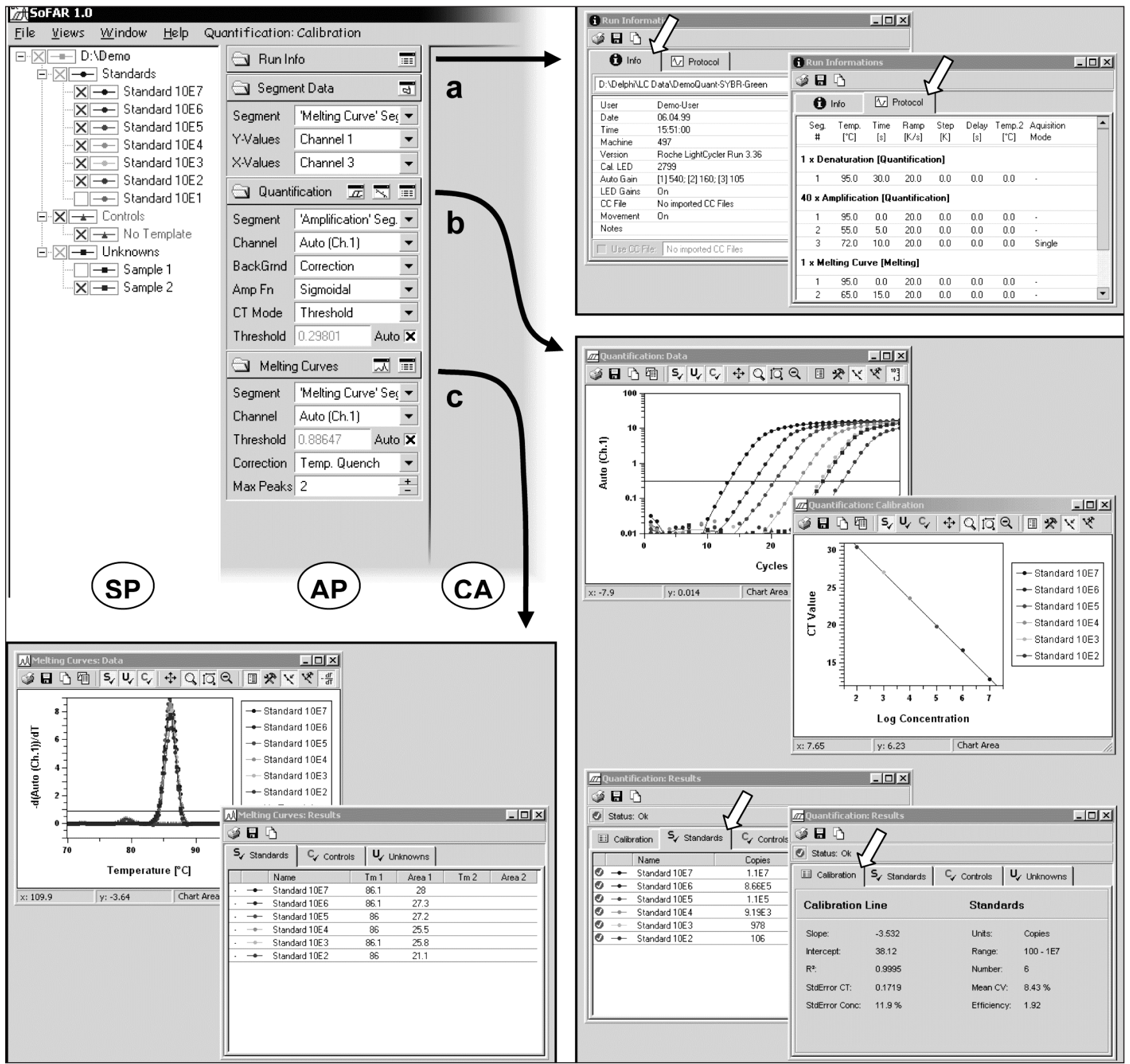

Figure 6. Overview of the application layout. The main window is shown as a section containing the selection panel (SP) and the adjustment panel (AP). The client windows for the presentation of data and results are shown in the client area (CA) of the application. (a) Client window of the Run Info section with both tabulator-sheets (see arrows) selected. (b) Client window of the Quantification section. The results window is shown with the selected tabulator-sheets "Standards" and "Calibration" (see arrows). (c) Client windows of the Melting Curves section. 
ing the kind of background correction (initial value subtraction, minimum value subtraction, and adaptive correction), the amplification functions used (exponential, logistic, sigmoid-exponential, and spline), and the method of $\mathrm{C}_{\mathrm{T}}$ calculation (noiseband, threshold, and second derivative maximum).

The Melting Curves section (c in Figure 6) contains one client window for the graphs of the data, either as melting curves or as melting peaks, and one client window for the $T_{m}$ and peak areas. The software allows one to define a threshold for peak detection and the maximum number of peaks per curve. The user can decide whether the data should be corrected for the temperature quench.

All results can be saved, printed, or copied to the Windows clipboard. The charts can be copied as scalable vector graphics (Windows-enhanced metafile format) or as tab-delimited text describing the data shown in the chart.

\section{CONCLUSIONS}

SoFAR is a standalone, easy-to-use software for the reliable and accurate evaluation of real-time PCR fluorescence data acquired with the LightCycler system. To our knowledge, it is the first software to apply algorithms for the adaptive correction of signal trends and fully automated detection and characterization of exponential phases in amplification curves. The precision of $\mathrm{C}_{\mathrm{T}}$ value calculations is increased by the introduction of a sigmoid function, fitted to the determined exponential phases and the adjacent points, ensuring an effective noise filtering. It is able to correct melting curve data for temperature-dependent quench, enhancing the dynamic range and sensitivity of melting curve analyses, required for reliable quantitative analyses of sequence variants with a high dynamic range.

\section{ACKNOWLEDGMENTS}

This work was supported by the Deutsche Forschungsgemeinschaft (Graduiertenkolleg Molekulare Biologie und Pharmakologie) and the Fonds der Chemischen Industrie. The authors thank Björn Tews, Christiane Thömmes and Annette Freist for "beta-testing" and Gunnar Wrobel for reading the manuscript.

\section{REFERENCES}

1.Bernard, P.S., G.H. Pritham, and C.T. Wittwer. 1999. Color multiplexing hybridization probes using the apolipoprotein E locus as a model system for genotyping. Anal. Biochem. 273:221-228.

2.Cardullo, R.A., S. Agrawal, C. Flores, P.C. Zamecnik, and D.E. Wolf. 1988. Detection of nucleic acid hybridization by nonradiative fluorescence resonance energy transfer. Proc. Natl. Acad. Sci. USA 85:8790-8794.

3.Craven, P. and G. Wahlba. 1979. Smoothing noisy data with spline functions. Numer. Math. 31:377-403.

4.de Boor, C. 2001. Calculation of the smoothing spline with weighted roughness measure. Math. Models Methods Appl. Sci. 11:33-41.

5.Heid, C.A., J. Stevens, K.J. Livak, and P.M. Williams. 1996. Real time quantitative PCR. Genome Res. 6:986-994.

6.Higuchi, R., C. Fockler, G. Dollinger, and R. Watson. 1993. Kinetic PCR analysis: realtime monitoring of DNA amplification reactions. Biotechnology 11:1026-1030.

7.Holland, P.M., R.D. Abramson, R. Watson, and D.H. Gelfand. 1991. Detection of specific polymerase chain reaction product by utilizing the $5^{\prime} \rightarrow 3^{\prime}$ exonuclease activity of Thermus aquaticus DNA polymerase. Proc. Natl. Acad. Sci. USA 88:7276-7280.

8.Kostrikis, L.G., S. Tyagi, M.M. Mhlanga, D.D. Ho, and F.R. Kramer. 1998. Spectral genotyping of human alleles. Science 279:1228-1229.

9.Liu, W. and D.A. Saint. 2002. A new method of real time reverse transcription polymerase chain reaction assay based on simulation of polymerase chain reaction kinetics. Anal. Biochem. 302:52-59.

10.Liu, W. and D.A. Saint. 2002. Validation of a quantitative method for real time PCR kinetics. Biochem. Biophys. Res. Commun. 294:347-353.

11.Livak, K.J. and T.D. Schmittgen. 2001. Analysis of relative gene expression data using real-time quantitative PCR and the 2(- $\Delta \Delta \mathrm{C}(\mathrm{T}))$ method. Methods 25:402-408.

12.Marquardt, D.W. 1963. An algorithm for least-square estimation for non-linear parameters. J. Soc. Ind. Appl. Math. 11:431-441.

13.Meuer, S., C. Wittwer, and K. Nakagawara (Eds.). 2000. Rapid Cycle Real Time PCRMethods And Applications. Springer-Verlag, Heidelberg, Germany.

14.Nazarenko, I.A., S.K. Bhatnagar, and R.J. Hohman. 1997. A closed tube format for amplification and detection of DNA based on energy transfer. Nucleic Acids Res. 25:25162521.

15.Peccoud, J. and C. Jacob. 1998. Statistical estimations of PCR amplification rates, p. 117. In F. Ferré (Ed.), Gene Quantification. Birkhäuser, New York.

16.Pfaffl, M.W., G.W. Horgan, and L.
Dempfle. 2002. Relative expression software tool (REST) for group-wise comparison and statistical analysis of relative expression results in real-time PCR. Nucleic Acids Res. 30:e36.

17.Rice, J. and M. Rosenblatt. 1983. Smoothing splines: regression, derivatives and deconvolution. Ann. Statistics 11:141-156.

18.Schnell, S. and C. Mendoza. 1997. Theoretical description of the polymerase chain reaction. J. Theor. Biol. 188:313-318.

19.Stolovitzky, G. and G. Cecchi. 1996. Efficiency of DNA replication in the polymerase chain reaction. Proc. Natl. Acad. Sci. USA 93:12947-12952.

20.Tyagi, S. and F.R. Kramer. 1996. Molecular beacons: probes that fluoresce upon hybridization. Nat. Biotechnol. 14:303-308.

21.Whitcombe, D., J. Theaker, S.P. Guy, T. Brown, and S. Little. 1999. Detection of PCR products using self-probing amplicons and fluorescence. Nat. Biotechnol. 17:804807.

22.Wittwer, C.T., K.M. Ririe, R.V. Andrew, D.A. David, R.A. Gundry, and U.J. Balis. 1997. The LightCycler ${ }^{\mathrm{TM}}$ : a microvolume multisample fluorimeter with rapid temperature control. BioTechniques 22:176-181.

23.Wittwer, C.T., M.G. Herrmann, A.A. Moss, and R.P. Rasmussen. 1997. Continuous fluorescence monitoring of rapid cycle DNA amplification. BioTechniques 22:130-138.

Received 3 September 2002; accepted 15 November 2002.

\section{Address correspondence to:}

Dr. Jochen Wilhelm

Institut für Biochemie, $\mathrm{FB} 08$

Justus-Liebig-Universität Giessen

Heinrich-Buff-Ring 58

D-35392 Giessen, Germany

e-mail: jochen.wilhelm@chemie.bio.

uni-giessen.de

For reprints of this or any other article, contact Reprints@BioTechniques.com 